\title{
THE AM Her PERIOD SPIKE
}

\author{
J.P. LASOTA \\ Département d'Astrophysique Relativiste et Cosmologie \\ Observatoire de Paris, Section de Meudon \\ 92195 Meudon Principal Cedex, France
}

ABSTRACT. The AM Her period spike is due to the resumption of mass transfer after evolving through the period gap. This implies that (i) at the spike period all secondary stars are the same (ii) most $\mathrm{AM}$ Her white dwarfs have a unique mass $\simeq 0.6-0.7 M_{\odot}$ (iii) nova explosions on magnetic white dwarfs remove exactly the mass accreted between outbursts (iv) the newly discovered AM Her system at $127 \mathrm{~min}$ has a mass $\simeq 1.3-1.4 M_{\odot}(\mathrm{v})$ the maximum magnetic field for most AM Her's is $\leq 4 \times 10^{7} \mathrm{G}$.

There is a large, highly significant (Morris et al. 1987) accumulation (6 out of 15) of AM Her systems in the period interval 113.5 - 114.8 minutes. Recently Hameury et al. (1988a) proposed an explanation for this in terms of the resumption of mass transfer after these systems have crossed the period gap. When accretion resumes at a period of about $2 \mathrm{hr}$ the fully convective secondary star undergoes a short (Kelvin-Helmholtz time) episode of adiabatic expansion, until it is sufficiently far out of thermal equilibrium that it can contract under the influence of mass loss. The orbital period first increases by 2 to $3 \mathrm{~min}$, reaches a maximum, and decreases. This enhances the discovery probability at this period: (i) the mass transfer rate $-\dot{M}_{2}$ is about twice the value for a star close to the main sequence contracting in response to mass loss, and (ii) the expansion and subsequent contraction of the secondary lengthens the time spent near the initial period. To give the observed period "spike" requires most systems to have similar parameters when mass transfer resumes at the lower edge of the period gap: otherwise the initial value of $P_{0}$ would not be the same and the spike would be smeared out. The secondary stars are naturally similar if the gap is caused (Rappaport et al. 1983, Spruit \& Ritter 1983) by them becoming fully convective. Thus at the spike, $M_{2} \simeq 0.2 \mathrm{M}_{\odot}$. Further, the period spike implies constraints on the primary masses which have been examined by Hameury et al. (1988b). Higher masses cause a narrower period gap and hence a higher value $P_{0}$ of the initial period below the gap. The value of the primary mass for systems at the period spike depends on the fate of the matter accreted during the secular evolution. Hameury et al. (1988c) show that for magnetic systems nova outbursts remove (almost) exactly the mass accreted between explosions. The white dwarf masses therefore stay constant. Using the fact that the newly discovered AM Her system EXO033319-2554.2 has a period of $127 \mathrm{~min}$ they conclude that most AM Her systems have a unique mass $\simeq 0.6-0.7 M_{\odot}$, while EXO033319-2554.2 has a significantly larger white dwarf mass 
$\left(\simeq 1.2-1.3 M_{\odot}\right)$. This prediction was subsequently supported by radial-velocity measurements (Bevermann et al. 1988).

Most AM Her binaries have white dwarfs magnetic fields (Schmidt et al 1986) $B_{1} \leq 3 \times 10^{7} \mathrm{G}$, whereas isolated white dwarfs can have fields up to at least $5 \times 10^{8} \mathrm{G}$ (Schmidt 1987). Since most properties of magnetic binaries depend on the magnetic moment, the upper limit on magnetic field has been difficult to understand. However, if one neglects the small class of massive systems (like EXO033319-2554.2) we may regard AM Her systems as having effectively a unique white dwarf mass. With $R_{1}$ essentially fixed at $\simeq 8 \times 10^{8} \mathrm{~cm}$, the limit on $\mathrm{B}$ becomes a limit on moments $\mu_{1}$ through $\mu_{1}=5 \times 10^{33} B_{7} \mathrm{G} \mathrm{cm}$ where $B_{7}$ is $B_{1}$ in units of $10^{7}$ G. Hameury et ai . (1988d) have shown that, moments $\mu_{1} \geq 2 \times 10^{34} \mathrm{G} \mathrm{cm}^{3}$, (i.e. fields stronger than $B_{7} \simeq 4$ ) imply catastrophic angular momentum losses $\dot{J}$ via magnetic braking involving the white dwarf magnetic field (Schmidt et al.1986). These drive the secular evolution so rapidly that the systems enter a prolonged period gap at $P \sim 5 \mathrm{hr}$, remaining as detached systems for a time of order the Hubble time. Some of these systems may in fact be observed as apparently isolated, rapidly rotating and highly magnetic white dwarfs (Schmidt 1987), or, for very old systems, as non-magnetic white dwarfs with brown dwarf companions.

\section{References}

Beuermann, K., Thomas, H.C. \& Schwope, A., 1988. Astr. Astrophys., 195, L15.

Hameury, J.M., King, A.R., Lasota, J.P. \& Ritter, H., 1988a. Mon. Not. R. astr. Soc., 231, 535.

Hameury, J.M., King, A.R. \& Lasota, J.P., 1988b. Astr. Astrophys., 195, L12.

Hameury, J .M., King, A.R., Lasota, J.P. \& Livio, M., 1988c. Mon. Not. R. astr. Soc., , submitted

Hameury, J .M., King, A.R. \& Lasota, J.P., 1988d. Mon. Not. R. astr. Soc., , .submitted Morris, S.L., Schmidt, G.D., Liebert, J., Stocke, J., Gioia, I. \& Maccacaro, T., 1987. Astrophys. $J ., 314,641$.

Rappaport, S., Verbunt, F. \& Joss, P.C., 1983. Astrophys. J., 275, 713.

Schmidt, G., Stockman, H.S. \& Grandi, S.A., 1986. Astrophys. J., 300, 804.

Schmidt, G., 1987. Mem. Soc. Astron. It. 209, 227.

Spruit, H.C. \& Ritter, H., 1983. Astr. Astrophys., 124, 267. 